\title{
МЕЖСЛОЕВАЯ САМОСБОРКА 2D НАНОКОМПОЗИТОВ НА ОСНОВЕ СЛОИСТЫХ ПРЕКУРСОРОВ
}

\author{
() 2017 Т. В. Куликова, А. В. Тучин, Д. А. Тестов, Л. А. Битюцкая \\ Воронежский государственный университет, Университетская пл., 1, 394018 Воронеж, Россия \\ e-mail:kaimt@mail.ru
}

Поступила в редакцию 14.07.2017 г.

\begin{abstract}
Аннотация. Экспериментально и теоретически исследованы условия формирования 2D аллотропов слоистых прекурсоров (сурьма, графит) и композитов на их основе межслоевой самосборкой из коллоидных растворов. Определены режимы возникновения самоактивированных коллоидных растворов изопропанол/сурьма, сопровождающихся наличием длинновременных нелинейных гидродинамических эффектов в коллоидном растворе, коррелирующих с периодическим изменением размеров частиц в растворе. Теоретически показано, что мультислои сурьмы отличаются по своим зарядовым свойствам, и наблюдаемые экспериментально процессы свидетельствуют о наличии в объеме раствора 2D структур с различным числом слоев, различающиеся типом и величиной заряда. Получены два типа композитных структур: многослойная структура мультиграфен/сурьма и полиморфная - мультиграфен/нановолокна.
\end{abstract}

Ключевые слова: композит, самосборка, слоистый прекурсор, коллоидный раствор, ультразвук, гидродинамика, неустойчивость, электростатика.

\section{ВВЕДЕНИЕ}

С момента открытия графена [1, 2] многократно возрос интерес мировой научной общественности к возможности создания аналогичных 2D материалов, и основной тенденцией современного материаловедения стало создание и изучение свойств 2D материалов, состоящих из одного или двух элементов III-VI группы периодической системы, а также композитов на их основе. При этом наиболее стабильные модификации 2D материалов формируются из группы пниктидов и в отличие от графена демонстрируют наличие ненулевой ширины запрещенной зоны, а также чувствительность электронной структуры к внешним деформациям [3-5]. Среди этой группы можно выделить сурьму, как тяжелый элемент с сильным спин-орбитальным взаимодействием, позволяющим наблюдать топологические эффекты, а также формировать стабильные 2D аллотропы [6, 7].

В настоящее время разработано множество методов получения графена и других 2D материалов, начиная от «нобелевского» механического отшелушивания от исходного слоистого кристаллического материала и заканчивая сложными и дорогостоящими методиками послойного эпитаксиального выращивания в случае, если исходные компонен- ты не образуют слоистых аллотропов. Для 2D аллотропа сурьмы - антимонена можно выделить три основные применяемые методики: механическое расслоение, жидкофазное расслоение и Ван-дерВаальсова эпитаксия. При механическом расслоении удается получать моно- и мультиантимонен с использованием буферного полимерного слоя, однако, основным недостатком метода остается непригодность для массового производства [8]. Увеличить выход материала удалось авторам работы [9], применив метод жидкофазного расслоения ультразвуком измельченных кристаллитов сурьмы. В результате эксперимента были получены нанолисты антимонена гексагональной симметрии с латеральными размерами порядка единиц микрон, поперечными размерами порядка единиц нанометров. Ван-дер-Ваальсова эпитаксия позволяет получать листы антимонена с латеральными размерами в диапазоне от 100 нм до 10 мкм и толщиной - от 1 до 50 нм [10]. Однако метод требует более сложного технического оснащения, а также наличия определенного типа подложки с отсутствием оборванных связей на поверхности для успешного формирования на ней листов антимонена. Таким образом, метод жидкофазного расслоения сегодня представляется наиболее перспективным для формирования 
2D аллотропов и композитов на их основе с точки зрения энергоэффективности и повышения полезного выхода материала.

Одним из наиболее перспективных композитных материалов для систем хранения энергии является композит сурьма/углерод, так как сурьма как электродный материал обладает большой удельной емкостью - 660 мАч/г, а углерод эффективно предотвращает деградацию материала [11-19]. Многие из методов получения этого композита длительные и энергозатратные, в связи с чем ведется активный поиск высокопроизводительного синтеза композита сурьма/углерод с характеристиками, превосходящими графитовые электроды.

Ранее нами предложена методика получения фазы карбида кремния в коллоидном растворе коротких углеродных нанотрубок и наноразмерного пиролитического диоксида кремния при комнатной температуре [20, 21].

Целью работы является изучение условий формирования композитных структур в коллоидных растворах слоистых прекурсоров (сурьма, графит).

\section{ТЕОРЕТИЧЕСКАЯ ЧАСТЬ}

Самосборкой называется процесс, при котором из отдельных компонентов или составляющих смеси образуется самопроизвольно упорядоченное единое целое благодаря минимизации их общей энергии. В отличие от молекулярных систем строительными блоками в коллоидных растворах слоистых прекурсоров являются 2D аллотропы. Коллоидный раствор сурьмы отвечает пяти принципам самосборки, сформулированных для молекулярных систем: наличие строительных блоков и их подвижности, обратимости, среды протекания процесса, межмолекулярных взаимодействий [22]. Но имеет принципиальное отличие - развитую 2D поверхность. Для изучения возможных взаимодействий строительных блоков - моно- и мультислоев в коллоидном растворе сурьмы проводилось квантово-химическое моделирование.

Квантово-химическое моделирование фрагментов слоев антимонена размером $5 \times 5$ элементарных ячеек, пассивированных с торцов водородом, проводилось теорией функционала электронной плотности (DFT). Антимонен представляет собой гофрированный бислой, состоящий из деформированных гексагонов, в вершинах которых располагаются атомы сурьмы. Расстояния между нейтральными атомами в пределах слоя $R=2.970 \AA$.

При последовательном увеличении числа слоев происходит качественное изменение распределения электронной плотности. Атомы двухслойного антимонена внешних слоев имеют избыточный эффективный положительный заряд 0.21 е, внутренние -0.21 е. Межатомное расстояние увеличивается до 2.978-2.979 А. Минимальное расстояние между атомами соседних слоев составляет $3.351 \AA$.

В трехслойном антимонене средний бислой почти нейтральный (эффективный заряд 0.009 е). Обнаружена асимметрия распределения заряда во внешних слоях: внешние грани сохраняют положительный заряд $Q_{\text {eff }}=0.147$ е, внутренние грани имеют заряд -0.156 е. Межатомные расстояния в среднем слое $2.978 \AA$, во внешних слоях $2.977 \AA$, что соответствует двухслойному антимонену.

Послойный рост определяет стабилизацию антимонена за счет увеличения числа межслоевых связей, однако величина приведенной энергии на слой уменьшается с $E_{\mathrm{bsh}}=0.734-0.499$ эВ/слой элементарной ячейки для двух- и трехслойной структуры. Данный результат согласуется с увеличением минимального расстояния между атомами соседних слоев в трехслойном антимонене до 3.590 Е.

Таким образом, перераспределение электронной плотности в многослойных структурах сурьмы определяет индуцирование избыточного положительного заряда на внешних гранях слоев. Внутренние слои нейтральные как в однослойном антимонене, при этом приведенная межслоевая энергия связи между внешними и внутренними слоями уменьшается почти в 1.5 раза, по сравнению с двухслойными структурами, в которых отсутствует нейтральный слой. В целом, последовательный рост числа слоев определяет стабилизацию многослойной сурьмы, однако предопределяет ее более легкое расщепление на слои за счет меньшей приведенной межслоевой энергии связи, наличия исходного положительного заряда на внешней грани (рис. 1, табл. 1).

Таким образом, наличие заряда в слоях является основанием для разработки методов и технологии самосборки планарных 2D структур слоистых прекурсоров и композитов на их основе.

\section{ЭКСПЕРИМЕНТАЛЬНАЯ ЧАСТЬ}

С целью получения планарных 2D структур сурьмы и графита и композитов на их основе использовалось многочасовое ультразвуковое воздействие на раствор изопропилового спирта и воды в соотношении 4:1 и мелкодисперсных монокристалла сурьмы марки Су000 в концентрации

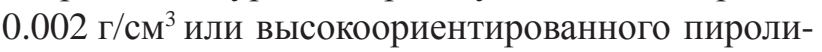

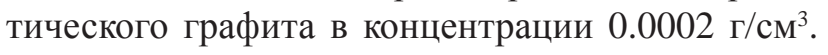



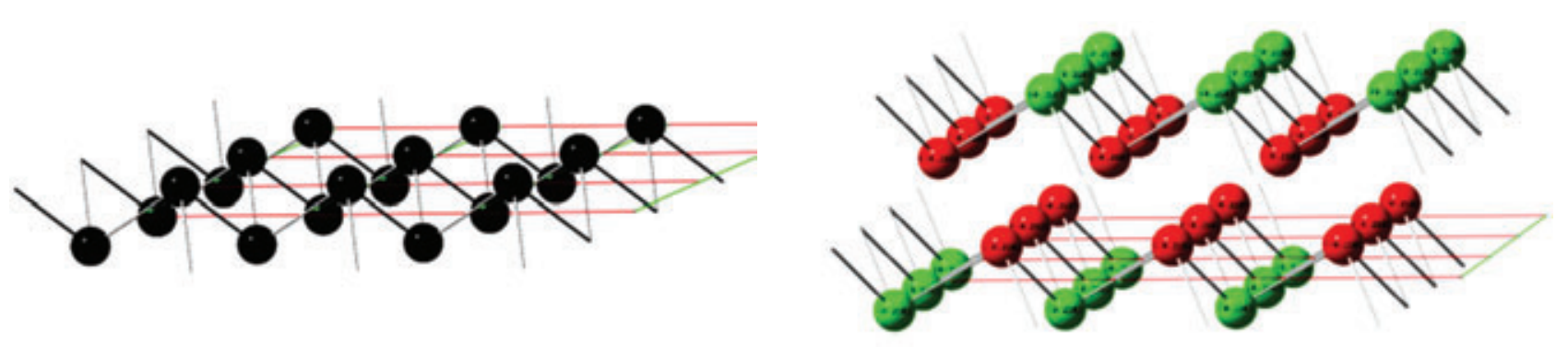

$a \quad b$
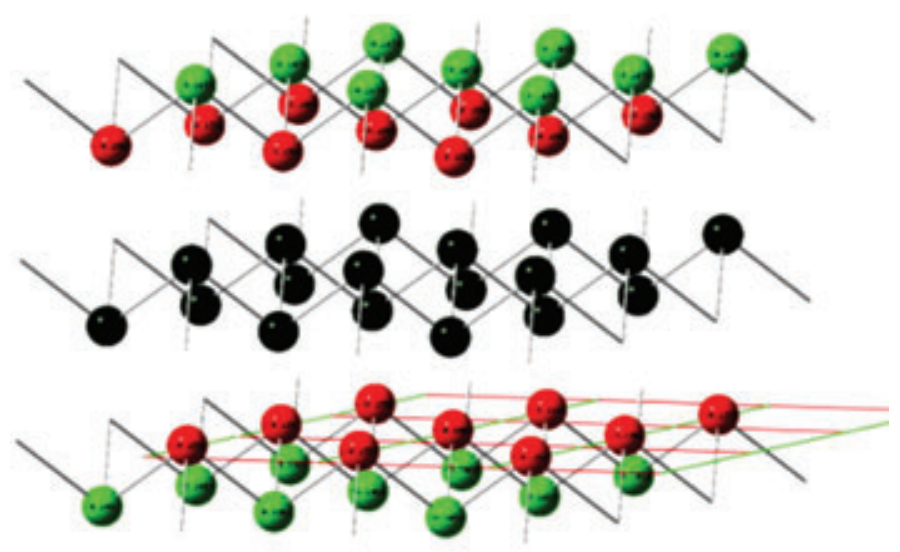

$c$

Рис. 1. Распределение эффективного заряда в антимонене в зависимости от числа слоев $n=1(a), 2(b)$ и 3 (c)

[Fig. 1. The distribution of the effective charge in antimonene, depending on the number of layers $n=1(a), 2(b)$ и $3(c)$ ]

Таблица 1. Кратчайшие внутрислоевые и межслоевые расстояния, приведенная энергия и эффективный заряд антимонена в зависимости от числа слоев

[Table 1. The shortest inside the layer and interlayer distances, the reduced energy and the effective charge of antimony, depending on the number of layers]

\begin{tabular}{|c|c|c|c|c|}
\hline$n$ & $R, \mathrm{E}$ & $R_{\mathrm{sh}}, \mathrm{E}$ & $\begin{array}{c}E_{\mathrm{b}}, \text { эВ/слой эл. ячейки } \\
{\left[E_{\mathrm{b}}, \mathrm{eV} / \text { layer of the unit cell }\right]}\end{array}$ & $Q_{\text {eff }}, \mathrm{e}$ \\
\hline 1 & 2.970 & - & - & 0 \\
\hline \multirow{4}{*}{2} & 2978 & \multirow{4}{*}{3.351} & \multirow{4}{*}{0.734} & 0.210 \\
\hline & 2.910 & & & -0.210 \\
\hline & 2978 & & & -0.210 \\
\hline & $2.9 / 8$ & & & 0.210 \\
\hline \multirow{6}{*}{3} & & \multirow{3}{*}{3.590} & \multirow{3}{*}{0.499} & 0.147 \\
\hline & 2.911 & & & -0.156 \\
\hline & \multirow{2}{*}{2.978} & & & 0.009 \\
\hline & & \multirow{3}{*}{3.590} & \multirow{3}{*}{0.499} & 0.009 \\
\hline & \multirow{2}{*}{2.977} & & & -0.156 \\
\hline & & & & 0.147 \\
\hline
\end{tabular}

Диспергирование проводилось воздействием ультразвука с частотой 22 кГц и мощностью 100 Вт с помощью диспергатора УЗД2-0.1/22.

Раствор подвергался ультразвуковой обработке в течение 3 часов для графита и 5 часов для сурьмы.

С целью получения композитов сурьма/углерод растворы после диспергации смешивались и диспергировались повторно в течение часа.
После диспергирования коллоидного раствора сурьмы с концентрацией 0.002 г $/ \mathrm{cm}^{3}$ наблюдались длинновременные нелинейные гидродинамические эффекты, что позволяет считать систему термодинамически устойчивой:

- образование вихрей в объеме коллоидного раствора, число которых зависело от концентрации и объема раствора (рис. 2); 


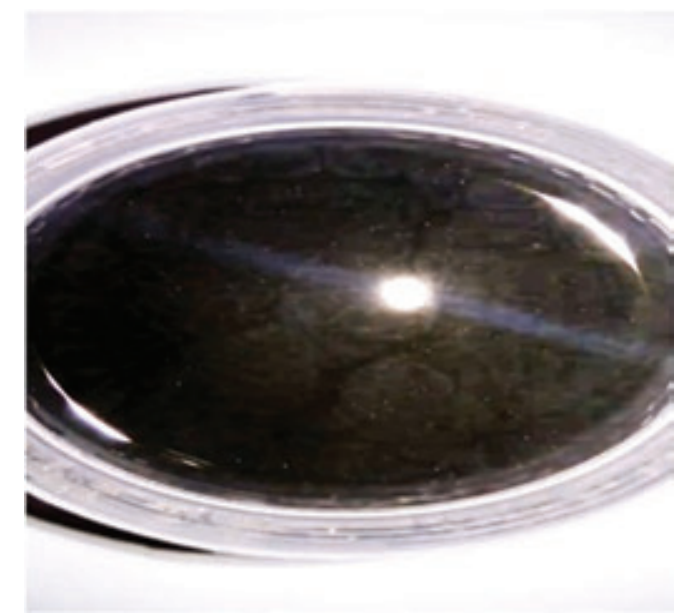

Рис. 2. Образование вихрей в объеме коллоидного раствора сурьмы после УЗ воздействия в течение 5 часов

[Fig. 2. The formation of vortices in the volume of the colloidal solution of antimony after ultrasonic exposure for 5 hours]

- колебательное изменение прозрачности раствора, происходящее в течение 5 часов после окончания диспергирования;

- образование на поверхности раствора динамической пленки сурьмы.

Наблюдаемые колебательные процессы коррелируют с периодическим изменением усредненных размеров частиц в коллоидном растворе в диапазоне $10^{2}-10^{5}$ нм, контролируемым методом динамического рассеяния света.

Во время нелинейных гидродинамических эффектов происходило взятие проб коллоидных растворов и осаждение на кремниевые монокристал-

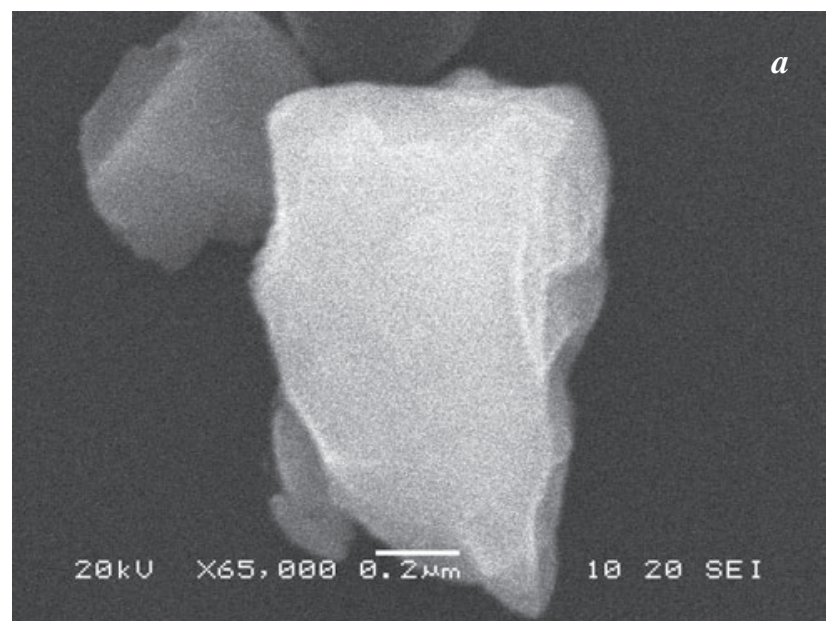

лические подложки с помощью капельной методики.

Для изучения морфологии полученных планарных структур использовалась сканирующая электронная микроскопия (СЭМ) - микроскоп Jeol JSM-6380LV в режиме вторичных электронов.

Многочасовое диспергирование коллоидного раствора сурьмы приводит к образованию планарных 2D структур различной толщины и латеральных размеров в диапазоне 0.5-10 мкм. В изученных режимах расслоения сурьмы до моноатомных слоев не зафиксировано.

В случае диспергирования коллоидного раствора графита расслоение происходит достаточно эффективно, причем в одном технологическом цикле формируются мультиграфен и полиморфная композитная структура - мультиграфен/нановолокна [23]. Композит представляет собой набор графеновых листов, содержащих на поверхности «клубок» нановолокон. Латеральные размеры композита $7 \times 2.5$ мкм, диаметр волокон составляет величину порядка 50 нм.

При диспергировании коллоидного раствора сурьмы и графита образуется многослойная композитная структура мультиграфен/сурьма с латеральными размерами порядка десятков микрон.

\section{ОБСУЖДЕНИЕ РЕЗУЛЬТАТОВ И ВЫВОДЫ}

В настоящей работе изучены закономерности формирования планарных 2D структур и композитов на их основе из коллоидных растворов изопропанол/сурьма и изопропанол/графит при ультра-

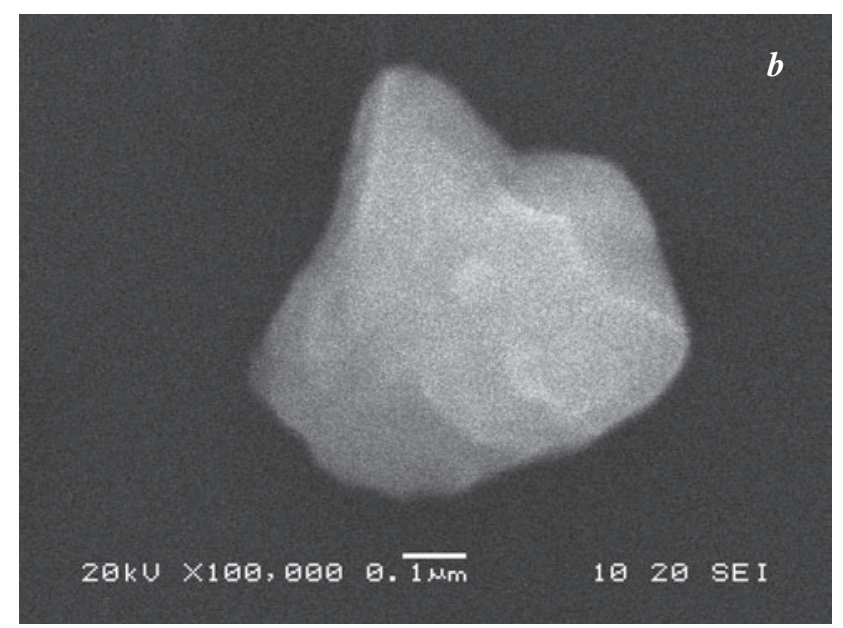

Рис. 3. Планарные структуры сурьмы, полученные в результате УЗ воздействия на коллоидный раствор изопропанол/сурьма в течение 5 часов

[Fig. 3. Planar structures of antimony obtained as a result of ultrasonic exposure on the colloidal solution of isopropanol/ antimony for 5 hours] 

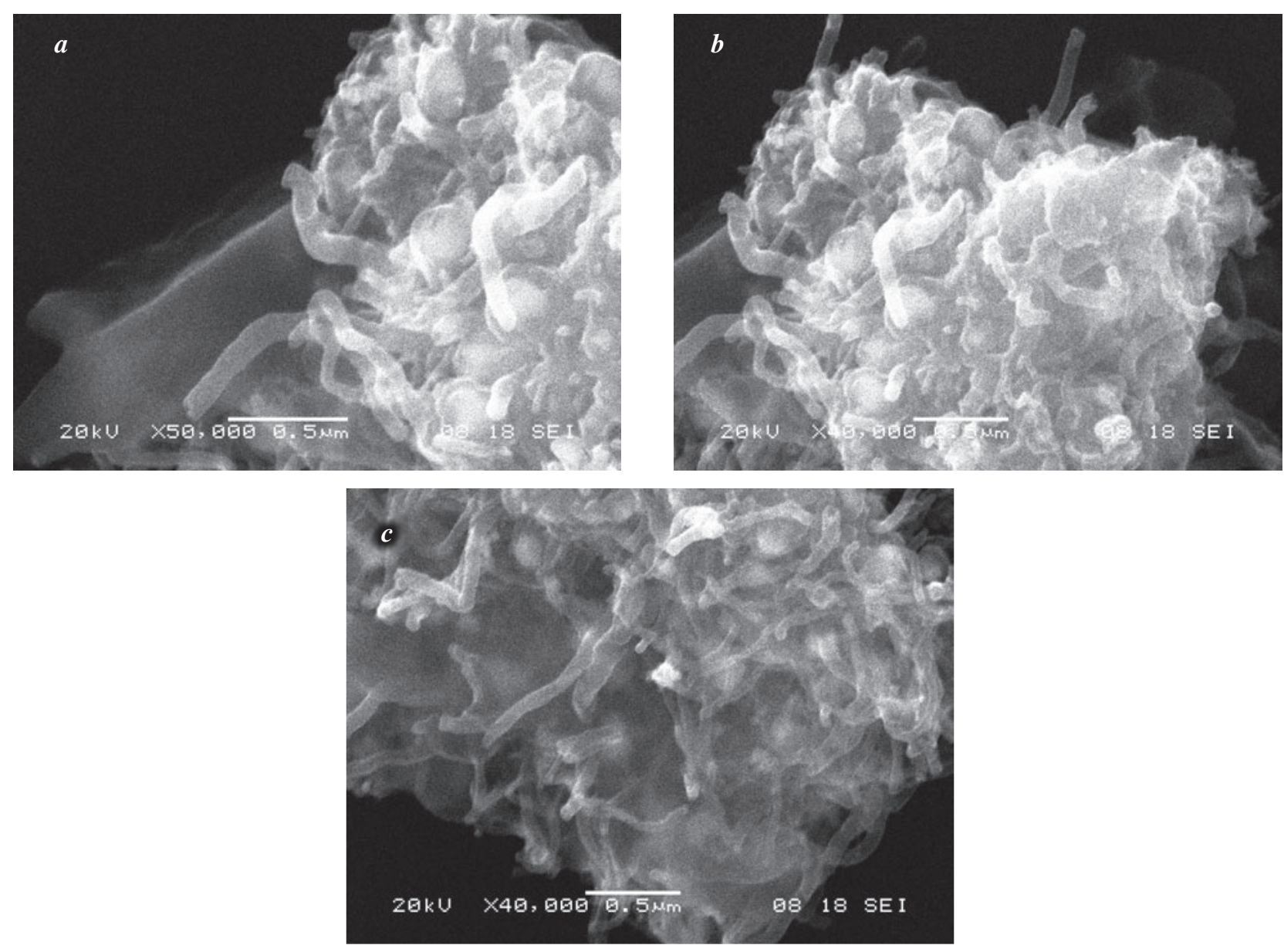

Рис. 4. СЭМ изображение композита мультиграфен/нановолокна, полученного при УЗ воздействии на коллоидный раствор изопропанол/графит в течение 3 часов

[Fig. 4. SEM image of a multigrafene/nanofiber composite obtained by ultrasonic exposure on the colloidal solution isopropanol/graphite for 3 hours]

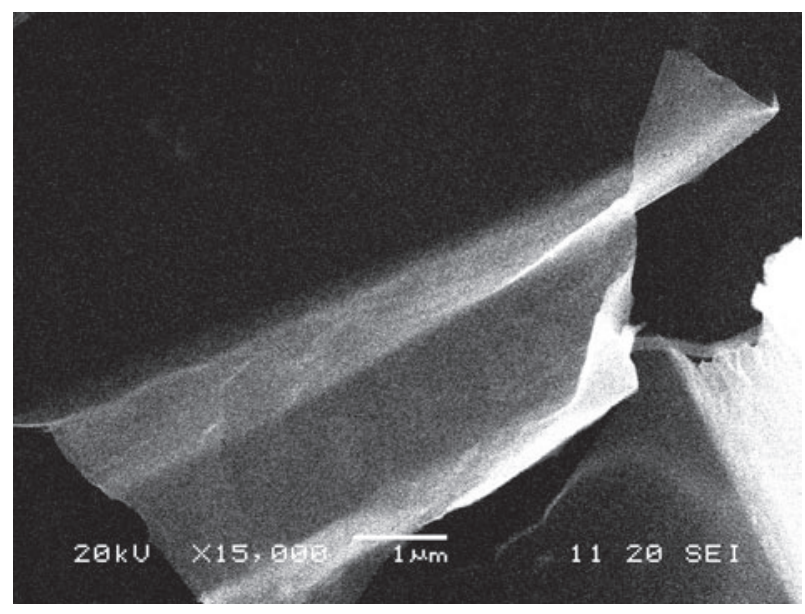

Рис. 5. СЭМ изображение мультиграфена, полученного при УЗ воздействии на коллоидный раствор изопропанол/графит в течение 3 часов

[Fig. 5. SEM image of a multigrafene obtained by ultrasonic exposure on the colloidal solution isopropanol/graphite for 3 hours] звуковом воздействии в нормальных условиях. Разработаны методики и оптимизированы режимы получения структур разного типа. Проведена морфологическая характеризация полученных мультислойных структур.

Экспериментально выявлены режимы возникновения самоактивированных коллоидных растворов, сопровождающихся наличием длинновременных гидродинамических эффектов, связанных с периодическим изменением размеров частиц в растворе. Теоретически показано, что мультислои сурьмы отличаются по своим зарядовым свойствам и наблюдаемые экспериментально процессы свидетельствуют о наличии в объеме раствора 2D структур с различным числом слоев, различающиеся типом и величиной заряда. Электростатически активная среда коллоидного раствора создает условия для самосборки как планарных структур исходных прекурсоров, так и композитных структур на их основе. 

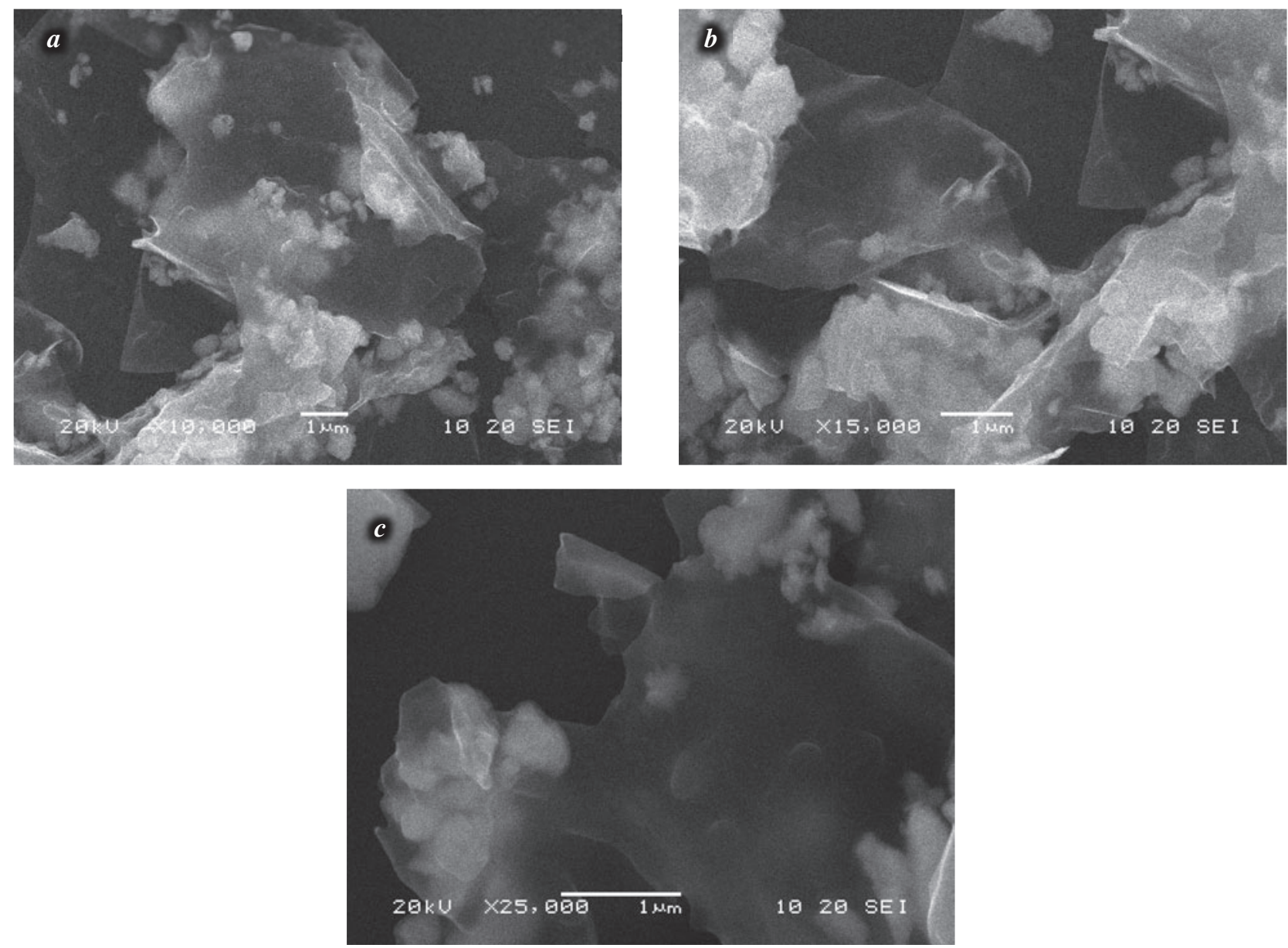

Рис. 6. СЭМ изображения композита мультиграфен/сурьма, полученного при УЗ воздействии на коллоидный раствор изопропанол/графит/сурьма в течение 1 часа

[Fig. 6. SEM image of a multigrafene/antimony composite obtained by ultrasonic exposure on the colloidal solution isopropanol/graphite / antimony for 1 hour]

В результате получены многослойные 2D аллотропы сурьмы и графита, а также два типа композитных структур: многослойная структура мультиграфен/сурьма и полиморфная - мультиграфен/нановолокна.

Работа выполнена при финансовой поддержке РФФИ (проект № 16-43-360281 p_a)

Выражаем благодарность Центру коллективного пользования научным оборудованием Воронежского государственного университета за активную поддержку работ молодых ученых!

\section{СПИСОК ЛИТЕРАТУРЫ}

1. Новоселов К. С. // УФН, 2011, № 181, с. 12991311.

2. Гейм А. К. // УФН, 2011, № 181, с. 1284-1298.

3. Yuanfeng Xu, Bo Peng, Hao Zhang, et al. // Ann. Phys. (Berlin), 2017, p. 1600152.

4. Shengli Zhang, Zhong Yan, Yafei Li, et al. // Angew. Chem. Int. Ed., 2015, № 54 (10), pp. 3112-3115.
5. Mingwen Zhao, Xiaoming Zhang, Linyang Li. // Scientific Reports, 2015, vol 5, p. 16108.

6. Bian G., Miller T., Chiang T.-C. // Physical Review Letters, 2011, № 107, vol. 3, p. 036802.

7. Sung Hwan Kim, Kyung-Hwan Jin, Joonbum Park, et al. // Scientific Reports, 2016, vol. 6, p. 33193.

8. Pablo Ares, Fernando Aguilar-Galindo, David Rodríguez-San-Miguel, et al. // Adv. Mater., 2016, №. 28 (30), pp. 6332-6336.

9. Carlos Gibaja, David Rodriguez-San-Miguel, Pablo Ares, et al. // Angew. Chem. Int. Ed., 2016, № 55 (46), pp. 14345-14349.

10. Jianping Ji, Xiufeng Song, Jizi Liu, et al. // Nature Communications, 2016, vol. 7, p. 13352.

11. Jiangfeng Qian, Yao Chen, Lin Wu, et al. // Chem. Commun., 2012, № 48, pp. 7070-7072.

12. Nithya C., Gopukumar S. // J. Mater. Chem. A, 2014, № 2, pp. 10516-10525.

13. Zhu Y., Han X., Xu Y., et al. // ACS Nano, 2013, № 7, vol. 7, pp. 6378-6386.

14. Zhou X., Zhong Y., Yang M., et al. // Chem. Commun., 2014, № 50, pp. 12888-12891. 
15. Hou H., Jing M., Yang Y., et al. // ACS Appl. Mater. Interfaces, 2014, № 6, vol. 18, pp. 16189-16196.

16. Zhou X., Dai Z., Bao J., Guo Y.-G. // J. Mater. Chem. A, 2013, № 1, pp. 13727-13731.

17. Wen Luo, Pengfei Zhang, Xuanpeng Wang, et al. // Journal of Power Sources, 2016, № 304 (340e345).

18. Fang Wan, Jin-Zhi Guo, Xiao-Hua Zhang, et al. // ACS Appl. Mater. Interfaces, 2016, № 8, vol. 12, pp. 77907799.

19. Ning Zhang, Yongchang Liu, Yanying Lu, et al. // Nano Res., 2015, № 8, p. 3384.
20. Тучин А. В., Жукалин Д. А., Битюцкая Л. А., Калашников А. В. // Письма о материалах, 2016, т. 6, № 4, с. 333-337.

21. Жукалин Д. А., Тучин А. В., Голощапов Д. Л., Битюцкая Л. А. // Письма в ЖТФ, 2015, т. 41, вып. 4, c. $1-6$.

22. Мансури Г. А. Принциипы нанотехнологий. М.: Научный мир, 2008, 320 с.

23. Wei Fan, Longsheng Zhang, Tianxi Liu. GrapheneCarbon Nanotube Hybrids for Energy and Environmental Applications. Springer Singapore, 2017, 104 p.

\title{
INTERLAYER SELF-ASSEMBLY OF 2D NANOCOMPOSITES BASED ON LAYERED PRECURSOR
}

\author{
(C) 2017 T. V. Kulikova, A. V. Tuchin, D. A. Testov, L. A. Bityutskaya \\ Voronezh State University, 1 Universitetskaya sq., 394018 Voronezh, Russia \\ e-mail: kaimt@mail.ru
}

Received 14.08.2017

\begin{abstract}
The purpose of this work is to study the conditions of formation of the composite structures in colloidal solutions of layered precursors (antimony, graphite).

Two-dimensional (2D) allotropes can be considered as building blocks in colloidal solutions of layered precursors. To study the possible interactions of building blocks - mono- and multilayers in the colloidal solution of antimony carried out quantum-chemical modeling by the density functional theory (DFT). With a consecutive increase of the number of layers in the structure of antimony, a qualitative change in the distribution of the electron density occurs and inducing of an excess positive charge on the outer faces of the layers. The inside layers are neutral as in a single-layer antimonene.

Thus, the presence of charge in the layers is the basis for developing methods and technology of the self-assembly of planar 2D structures of layered precursors and composites based on them.

The formation of the planar 2D structures of antimony and graphite and composites based on them occurs as a result of many hours of ultrasonic exposure to a solution of isopropyl alcohol and water and a finely dispersed single crystal of antimony or highly oriented pyrolytic graphite. The regimes of the occurs of self-activated colloidal solutions are determined. The self-activated colloidal solutions are characterized by the presence of long-term nonlinear hydrodynamic effects in a colloidal solution of the antimony, correlated with periodic changes in the particle size in the solution. The experimentally observed processes indicate of the presence of 2D structures with a different number of layers in the volume of the solution, differing in the type and magnitude of the charge.

The electrostatically active environment of the colloidal solution creates conditions for the self-assembly of the planar structures of the original precursors and composite structures based on them.

As a result, multilayered 2D allotropes of antimony, graphite and two types of composite structures: a multilayer multigrahene / antimony structure and a polymorphic - multigrafene / nanofiber were obtained.
\end{abstract}

Keywords: composite, self-assembly, layered precursor, colloidal solution, ultrasound, hydrodynamics, instability, electrostatics.

\section{ACKNOWLEDGMENTS}

The reported study was funded by RFBR according to the research(project No. 16-43-360281 p_a)

The research results were obtained with equipment of Voronezh State University Centre for Collective Use of Scientific Equipment.

\section{REFERENCES}

1. Novoselov K. S. UFN, 2011, no. 181, pp. 1299-1311. DOI: 10.3367/UFNr.0181.201112f.1299

2. Geim A. K. UFN, 2011, no. 181, pp. 1284-1298. DOI: 10.3367/UFNr.0181.201112e.1284

3. Yuanfeng Xu, Bo Peng, Hao Zhang, et al. Ann. Phys. (Berlin), 2017, p. 1600152. DOI: 10.1002/andp.2016 00152 
4. Shengli Zhang, Zhong Yan, Yafei Li, et al. Angew. Chem. Int. Ed., 2015, no. 54, vol. 10, pp. 3112-3115. DOI: 10.1002/anie.201411246

5. Mingwen Zhao, Xiaoming Zhang, Linyang Li. Scientific Reports, 2015, vol. 5, p. 16108. DOI: 10.1038/ srep16108

6. Bian G., Miller T., Chiang T.-C. Physical Review Letters, 2011, no. 107, vol. 3, p. 036802. DOI: 10.1103/ PhysRevLett.107.036802

7. Sung Hwan Kim, Kyung-Hwan Jin, Joonbum Park, et al. Scientific Reports, 2016, vol. 6, p. 33193. DOI: 10.1038/srep33193

8. Pablo Ares, Fernando Aguilar-Galindo, David Rodríguez-San-Miguel, et al. Adv. Mater., 2016, no. 28, vol. 30, pp. 6332-6336. DOI: 10.1002/adma.201602128

9. Carlos Gibaja, David Rodriguez-San-Miguel, Pablo Ares, et al. Angew. Chem. Int. Ed., 2016, no. 55, vol. 46, pp. 14345-14349. DOI: 10.1002/anie.201605298

10. Jianping Ji, Xiufeng Song, Jizi Liu, et al. Nature Communications, 2016, vol 7, p. 13352. DOI: 10.1038/ ncomms13352

11. Jiangfeng Qian, Yao Chen, Lin Wu, et al. Chem. Commun., 2012, no. 48, pp. 7070-7072. DOI: 10.1039/ C2CC32730A

12. Nithya C., Gopukumar S. J. Mater. Chem. A, 2014, no. 2, pp. 10516-10525. DOI: 10.1039/C4TA01324G

13. Zhu Y., Han X., Xu Y., et al. ACS Nano, 2013, no. 7, vol. 7, pp. 6378-6386. DOI: 10.1021/nn4025674
14. Zhou X., Zhong Y., Yang M., et al. Chem. Commun., 2014, no. 50, pp. 12888-12891. DOI: 10.1039/C4CC0 5989A

15. Hou H., Jing M., Yang Y., et al. ACS Appl. Mater. Interfaces, 2014, no. 6, vol. 18, pp. 16189-16196. DOI: 10. 1021/am504310k

16. Zhou X., Dai Z., Bao J., Guo Y.-G. J. Mater. Chem. A, 2013, no. 1, pp. 13727-13731. DOI: 10.1039/C3TA 13438E

17. Wen Luo, Pengfei Zhang, Xuanpeng Wang, et al. Journal of Power Sources, 2016, no. 304 (340e345). DOI: 10.1016/j.jpowsour.2015.11.047

18. Fang Wan, Jin-Zhi Guo, Xiao-Hua Zhang, et al. ACS Appl. Mater. Interfaces, 2016, no. 8, vol. 12, pp. 7790-7799. DOI: 10.1021/acsami.5b12242

19. Ning Zhang, Yongchang Liu, Yanying Lu, et al. Nano Res., 2015, vol. 8, p. 3384. DOI:10.1007/s12274-0150838-3

20. Tuchin A. V., Zhukalin D. A., Bityutskaya L. A., Kalashnikov A. V. Letters on Materials, 2016, no. 6, vol. 4, pp. 333-337

21. Zhukalin D. A., Tuchin A. V., Goloshchapov D. L., Bityutskaya L. A. Technical Physics Letters, 2015, no. 41, vol. 4, pp. 1-6.

22. Mansoori G. A. Principles of Nanotechnology. World Scientific Publishing Co. Pte. Ltd., 2005, 360 p.

23. Wei Fan, Longsheng Zhang, Tianxi Liu. GrapheneCarbon Nanotube Hybrids for Energy and Environmental Applications. Springer Singapore, 2017, 104 p. DOI: 10.1007/978-981-10-2803-8
Куликова Татьяна Валентиновна - аспирант кафедры физики полупроводников и микроэлектроники, Воронежский государственный университет; тел.: +7 (908) 1445155, e-mail: kaimt@mail.ru

Тучин Андрей Витальевич - к. ф.-м. н., доцент кафедры физики полупроводников и микроэлектроники, Воронежский государственный университет; тел.: +7 (908) 1485775, e-mail: a.tuchin@bk.ru

Тестов Дмитрий Александрович - студент кафедры физики полупроводников и микроэлектроники, Воронежский государственный университет; тел.: +7 (910) 2406971, e-mail: dmitriytestov@gmail.com

Битюикая Лариса Александровна - к. х. н., доцент кафедры физики полупроводников и микроэлектроники, Воронежский государственный университет; тел.: +7 (473) 2208481, e-mail: me144@phys.vsu.ru
Kulikova Tatyana V. - postgraduate student, Department of Physics of Semiconductors and Microelectronics, Voronezh State University; ph.: +7 (908) 1445155, e-mail: kaimt@mail.ru

Tuchin Andrey V. - Cand. Sci. (Phys.-Math.), Department of Physics of Semiconductors and Microelectronics, Voronezh State University; ph.: +7 (908) 1485775, e-mail: a.tuchin@bk.ru

Testov Dmitriy A. - student of Department of Physics of Semiconductors and Microelectronics, Voronezh State University; ph.: +7 (910) 2406971, e-mail: dmitriytestov@ gmail.com

Bityutskaya Larisa A. - Cand. Sci. (Chem.), Department of Physics of Semiconductors and Microelectronics, Voronezh State University; ph.: +7 (473) 2208481, e-mail: me144@phys.vsu.ru 